\title{
DISCRETIZED STABILITY AND ERROR GROWTH OF THE NONAUTONOMOUS PANTOGRAPH EQUATION*
}

\author{
CHENGMING HUANG ${ }^{\dagger}$ AND STEFAN VANDEWALLE ${ }^{\ddagger}$
}

\begin{abstract}
This paper is concerned with the stability properties of Runge-Kutta methods for the pantograph equation, a functional differential equation with a proportional delay. The focus is on nonautonomous equations. Both linear and nonlinear cases are considered. Sufficient and necessary conditions for the asymptotic stability of the numerical solution of general neutral pantograph equations are given. An upper bound for the error growth is investigated for algebraically stable methods applied to nonneutral equations. Finally, some stability results are extended to the case of a more general class of equations.
\end{abstract}

Key words. pantograph equation, asymptotic stability, error growth, Runge-Kutta methods

AMS subject classifications. 65L06, 65L07, 65L20

DOI. $10.1137 /$ S0036142902419296

1. Introduction. Many real-world phenomena can be modelled by initial value problems for functional differential equations of the form

$$
y^{\prime}(t)=f\left(t, y(t), y(t-\tau(t)), y^{\prime}(t-\tau(t))\right) .
$$

In recent years, the study of numerical solvers for this problem has attracted the attention of many authors. The classical case where the term $\tau(t)$ is a constant can be regarded as a representative of finite time delay and has been widely studied in the literature (see, for example, Baker [1], Bellen and Zennaro [5] and the extensive bibliography therein). Another interesting case, which can be viewed as a representative of infinite time delay, is that of the pantograph equation, where

$$
\tau(t)=(1-q) t, \quad q \in(0,1)
$$

For applications of this type of equation, we refer to Iserles [16].

In order to get insight into the stability of numerical methods for the pantograph equation, the scalar linear autonomous equation

$$
y^{\prime}(t)=a y(t)+b y(q t)+c y^{\prime}(q t), \quad t>0,
$$

has been used as a test problem and many interesting results have been found (cf. [2, $6,7,8,9,17,18,22,24,25])$. In the early work, a constant stepsize was considered. As pointed out in Liu [22, 24], however, this kind of stepsize precludes long time integration due to computer memory restrictions. In order to overcome this difficulty, Liu [22] transformed (1.2) into a differential equation with a constant delay by a change of variable, suggested by Jackiewicz [20]. Later, Liu [24] and Bellen,

${ }^{*}$ Received by the editors December 10, 2002; accepted for publication (in revised form) December 19, 2003; published electronically February 25, 2005. This research was funded by the Research Council of the K. U. Leuven through fellowship grant F/02/060 and by the NSF of China through project 10101027 .

http://www.siam.org/journals/sinum/42-5/41929.html

${ }^{\dagger}$ Department of Mathematics, Huazhong University of Science and Technology, Wuhan 430074, China (chengming_huang@hotmail.com).

${ }_{\ddagger}^{\ddagger}$ Katholieke Universiteit Leuven, Department of Computerscience, Celestijnenlaan 200A, B3001 Leuven, Belgium (Stefan.Vandewalle@cs.kuleuven.ac.be). 
Guglielmi, and Torelli [2] proposed nonconstant stepsize strategies where the stepsizes are geometrically increasing and they investigated the stability of $\theta$-methods. Recently, Koto [21] further studied the stability of general Runge-Kutta methods for the multidimensional system

$$
u^{\prime}(t)=e^{t} L u(t)+e^{t} M u(t+\log q)+N u^{\prime}(t+\log q), \quad t>0,
$$

which is obtained from the equation

$$
y^{\prime}(t)=L y(t)+M y(q t)+q N y^{\prime}(q t), \quad t>0,
$$

by a change of the independent variable $u(t)=y\left(e^{t}\right)$, where $L, M$, and $N$ are constant complex $d \times d$ matrices. In an abstract sense the geometrically increasing mesh approach and the exponential transform method may be considered to be essentially the same (cf. [21]). Relevant to the nonautonomous pantograph equation, however, only few results on numerical stability have been published. Bellen, Guglielmi, and Torelli [2], and Guglielmi and Zennaro [13] discussed the asymptotic stability of $\theta$ methods for scalar equations with variable coefficients. Bellen, Maset, and Torelli [4] studied the "first step" integration of linear systems of neutral type and investigated the contractivity of continuous Runge-Kutta methods. Zhang and Sun [28, 29] recently obtained some stability results of Runge-Kutta methods for a class of nonlinear equations of nonneutral type (see Theorem 6.1 of this paper).

In this paper, a new approach for proving numerical stability is introduced. Sufficient and necessary conditions for asymptotic stability are derived for both linear and nonlinear problems of neutral type. Also, upper bounds for the error growth are studied for nonneutral systems and some sharper results than those published in the literature are obtained.

This paper is organized as follows. In section 2, the discrete schemes based on Runge-Kutta methods are introduced. In section 3, we focus on the asymptotic stability of the schemes for linear systems of neutral type with variable coefficients. In section 4, an upper bound for the error growth is given for a class of linear problems. In section 5, we turn our attention to nonlinear equations and an asymptotic stability result is derived. In section 6 , we further investigate the error growth bound for a class of nonlinear problems. In section 7 , we generalize our stability analysis to the case of a more general class of equations. Finally, in section 8, some conclusions are drawn.

2. Adaptation of Runge-Kutta methods to functional-differential equations of pantograph type. In this section, we consider the adaptation of RungeKutta methods to the pantograph equation

$$
\left\{\begin{array}{l}
y^{\prime}(t)=f\left(t, y(t), y(q t), y^{\prime}(q t)\right), \quad t>0 \\
y(0)=y_{0}
\end{array}\right.
$$

where $f:[0,+\infty) \times \mathbb{C}^{d} \times \mathbb{C}^{d} \times \mathbb{C}^{d} \rightarrow \mathbb{C}^{d}$, is a given mapping and $q$ is a constant satisfying $q \in(0,1)$.

Let $(A, b, c)$ denote a given Runge-Kutta method characterized by the $s \times s$ matrix $A=\left(a_{i j}\right)$ and vectors $b=\left(b_{1}, \ldots, b_{s}\right)^{T}, c=\left(c_{1}, \ldots, c_{s}\right)^{T}$. In this paper we always assume that $\sum_{j=1}^{s} b_{j}=1$. Let $t_{n}, n=0,1, \ldots$, be grid points satisfying

$$
0=t_{0}<t_{1}<t_{2}<\cdots<\infty, \quad \lim _{n \rightarrow \infty} t_{n}=\infty,
$$


and $h_{n}=t_{n+1}-t_{n}$, the corresponding stepsizes. Approximations $y_{n+1}$ to $y\left(t_{n+1}\right)$ are defined by the following equations:

$$
\begin{aligned}
& Y_{i}^{(n)}=y_{n}+h_{n} \sum_{j=1}^{s} a_{i j} f\left(t_{n}+c_{j} h_{n}, Y_{j}^{(n)}, \bar{Y}_{j}^{(n-m)}, \hat{Y}_{j}^{(n-m)}\right), \quad i=1, \ldots, s \\
& y_{n+1}=y_{n}+h_{n} \sum_{j=1}^{s} b_{j} f\left(t_{n}+c_{j} h_{n}, Y_{j}^{(n)}, \bar{Y}_{j}^{(n-m)}, \hat{Y}_{j}^{(n-m)}\right),
\end{aligned}
$$

where each $Y_{j}^{(n)}$ is an approximation to $y\left(t_{n}+c_{j} h_{n}\right)$, the arguments $\bar{Y}_{j}^{(n-m)}$ and $\hat{Y}_{j}^{(n-m)}$ denote approximations to $y\left(q\left(t_{n}+c_{j} h_{n}\right)\right)$ and $y^{\prime}\left(q\left(t_{n}+c_{j} h_{n}\right)\right)$, respectively, obtained by specific interpolation procedures at the point $t=q\left(t_{n}+c_{j} h_{n}\right)$, and $m$ is a positive integer that will be defined later.

In this paper, we consider a nonconstant stepsize strategy where the stepsizes are geometrically increasing. This kind of grid was proposed by Liu [24], and by Bellen, Guglielmi, and Torelli [2]. As pointed out in the above references, it has two advantages. First, it can avoid the computer memory problems in the case of longtime integration. Second, an interpolation procedure is not necessary if we choose the grid such that every delayed point maps exactly onto a past grid point.

To formulate the grid, we partition the half-line $[0,+\infty)$ into a union of bounded intervals as follows:

$$
[0,+\infty)=[0, h] \bigcup_{k=0}^{\infty}\left(q^{-k} h, q^{-k-1} h\right],
$$

where $h$ is an arbitrary but fixed positive number. Second, we further divide every interval $\left(q^{-k} h, q^{-k-1} h\right]$ into a fixed number $m$ of subintervals whose length is proportionally increasing with the factor $p=q^{\frac{-1}{m}}$, i.e.,

$$
\left(q^{-k} h, q^{-k-1} h\right]=\bigcup_{i=1}^{m}\left(q^{-k} p^{i-1} h, q^{-k} p^{i} h\right] .
$$

The first interval $[0, h]$ is divided as follows:

$$
[0, h]=[0, q h] \bigcup_{i=1}^{m}\left(q p^{i-1} h, q p^{i} h\right] .
$$

Therefore, we obtain the global grid defined by

$$
t_{n}=p^{n-m-1} h, \quad n=1,2, \ldots,
$$

which gives

$$
h_{n}=t_{n+1}-t_{n}=p^{n-m-1}(p-1) h, \quad n=1,2, \ldots,
$$

and $h_{0}=q h$. Hence, for $n=m+1, m+2, \ldots$,

$$
q t_{n}=t_{n-m} \quad \text { and } \quad q h_{n}=h_{n-m},
$$

which shows the delayed point is just on the past grid point and

$$
q\left(t_{n}+c_{j} h_{n}\right)=t_{n-m}+c_{j} h_{n-m} .
$$


Therefore, we can set

$$
\begin{aligned}
& \bar{Y}_{j}^{(n-m)}=Y_{j}^{(n-m)}, \\
& \hat{Y}_{j}^{(n-m)}=f\left(t_{n-m}+c_{j} h_{n-m}, Y_{j}^{(n-m)}, Y_{j}^{(n-2 m)}, \hat{Y}_{j}^{(n-2 m)}\right),
\end{aligned}
$$

which on substitution into $(2.2)-(2.3)$ gives

$$
\begin{aligned}
Y_{i}^{(n)} & =y_{n}+h_{n} \sum_{j=1}^{s} a_{i j} \hat{Y}_{j}^{(n)}, \quad i=1, \ldots, s, \\
\hat{Y}_{j}^{(n)} & =f\left(t_{n}+c_{j} h_{n}, Y_{j}^{(n)}, Y_{j}^{(n-m)}, \hat{Y}_{j}^{(n-m)}\right), \quad j=1, \ldots, s, \\
y_{n+1} & =y_{n}+h_{n} \sum_{j=1}^{s} b_{j} \hat{Y}_{j}^{(n)} .
\end{aligned}
$$

Remark 2.1. In Liu [24], this kind of stepsize was used in the numerical examples although a slightly more general assumption on the grid was considered in the theoretical analysis. In Bellen, Guglielmi, and Torelli [2], the stepsize strategy in the theoretical analysis is that every interval $\left(q^{-k} h, q^{-k-1} h\right]$ is divided into $m$ intervals of the same size. The strategy of proportionally increasing stepsizes was also suggested in Remark 5.1 of their paper where it is pointed out that this choice can simplify the implementation of the method considerably and leads to a more regular behavior of the error.

Remark 2.2. Because we are interested in the stability of the numerical solution, we assume that the initial values $Y_{j}^{(n-1)}, \hat{Y}_{j}^{(n-1)}$, and $y_{n}$ are available for $n=1,2, \ldots, m$. For the integration of the first steps, i.e., the initializing methods, we refer to the paper by Bellen, Maset, and Torelli [4].

Now we introduce some concepts which will be used later.

Definition 2.3 (see [14]). The stability function of a Runge-Kutta method $(A, b, c)$ is defined by

$$
R(z)=1+z b^{T}\left(I_{s}-z A\right)^{-1} e,
$$

where $e=[1, \ldots, 1]^{T}$ and $I_{s}$ stands for the $s \times s$ identity matrix.

Definition 2.4. When $A$ is nonsingular, the method is called strictly stable at infinity if

$$
|R(\infty)|=\left|1-b^{T} A^{-1} e\right|<1 .
$$

Definition 2.5 (see [10]). A Runge-Kutta method $(A, b, c)$ is called algebraically stable if the following matrix $\mathcal{M}=\left[\mathcal{M}_{i j}\right]$ is nonnegative definite:

$$
\mathcal{M}=B A+A^{T} B-b b^{T}
$$

where $B=\operatorname{diag}\left(b_{1}, b_{2}, \ldots, b_{s}\right)$.

3. Linear stability. In this section, we discuss the stability of Runge-Kutta methods for linear systems of the form

$$
y^{\prime}(t)=L(t) y(t)+M(t) y(q t)+N(t) y^{\prime}(q t), \quad t>0,
$$

where $L(t), M(t)$, and $N(t)$ are complex $d \times d$ matrices whose entries are continuous functions. First, we recall some results on the stability of the analytical solution. For 
the autonomous case (1.4) of (3.1), a result obtained by Liu [23] (see also [16]) implies the following proposition.

Proposition 3.1. The zero solution of (1.4) is asymptotically stable if $L, M$ satisfy

$$
\sigma[L] \subset \mathbb{C}^{-}, \quad \rho\left[L^{-1} M\right]<1,
$$

where $\mathbb{C}^{-}=\{z \in \mathbb{C}: \operatorname{Re} z<0\}$, and $\sigma[\cdot]$ and $\rho[\cdot]$ denote the spectrum and spectral radius of a matrix, respectively.

For the asymptotic stability of the nonautonomous neutral system (3.1), a result obtained by Iserles and Terjeki [19] implies the following proposition.

Proposition 3.2. The zero solution of (3.1) is asymptotically stable if there exists a vector norm $\|\cdot\|_{*}$ on $\mathbb{C}^{d}$, with induced matrix norm and the corresponding logarithmic norm $\mu[\cdot]$ such that for all $t \geq 0$ the following statements hold:

$$
\begin{gathered}
\mu[L(t)] \leq 0, \quad\|N(t)\|_{*} \leq \xi_{0}<1, \quad \int_{0}^{\infty} \mu[L(t)] d t=-\infty \\
\max _{x \in[0, t]}\|M(x)+N(x) L(q x)\|_{*}+k_{0} \mu[L(t)]\left(1-\xi_{0}\right) \leq 0 \text { for some } k_{0} \in(0,1) .
\end{gathered}
$$

Now we analyze the conditions of Proposition 3.2 in order to motivate our assumptions for the numerical stability analysis. We consider the nonneutral case, i.e., $N(t)=0$. If $\limsup _{t \rightarrow \infty} \mu[L(t)]=0$, from (3.4) it follows that $M(t)=0, t \in[0, \infty)$, which leads to a trivial case. Therefore, we assume that for sufficiently large $t$, $\mu[L(t)] \leq L_{0}<0$, which implies that the matrix $L(t)$ is nonsingular. Hence, from the properties of the logarithmic norm (cf. [12]), it follows that

$$
\left\|L^{-1}(t)\right\|_{*} \leq \frac{1}{-\mu(L(t))} \leq \frac{1}{-L_{0}} .
$$

In addition, for every $u \in \mathbb{C}^{d}$,

$$
-\mu[L(t)]\|u\|_{*} \leq\|L(t) u\|_{*},
$$

which guarantees that, for every $v \in \mathbb{C}^{d}$,

$$
-\mu[L(t)]\left\|L^{-1}(t) M(t) v\right\|_{*} \leq\|M(t) v\|_{*} .
$$

Therefore, we have

$$
-\mu[L(t)]\left\|L^{-1}(t) M(t)\right\|_{*} \leq\|M(t)\|_{*},
$$

which, combined with (3.4), implies

$$
\left\|L^{-1}(t) M(t)\right\|_{*} \leq k_{0}<1 .
$$

In our analysis of the asymptotic stability of numerical methods, we will make use of the following assumption, which is a natural extension of the conditions for scalar equations given by Guglielmi and Zennaro [13], and which can also cover condition (3.2) in the autonomous case.

Assumption $\mathcal{A}$ : There exist a vector norm $\|\cdot\|_{*}$ on $\mathbb{C}^{d}$ and induced matrix norm such that the matrices $L(t), M(t)$, and $N(t)$ satisfy for all $t>0$

$$
\left\|L^{-1}(t)\right\|_{*} \leq C_{0}, \quad\left\|L^{-1}(t) N(t)\right\|_{*} \leq \hat{C}_{0}, \quad\left\|L^{-1}(t) M(t)\right\|_{*} \leq k_{0}<1,
$$


where $C_{0}, \hat{C}_{0}$ and $k_{0}$ are constants.

The application of method (2.4-2.6) to (3.1) leads to the following difference equation:

$$
\begin{aligned}
Y_{i}^{(n)}= & y_{n}+h_{n} \sum_{j=1}^{s} a_{i j} \hat{Y}_{j}^{(n)}, \quad i=1, \ldots, s, \\
\hat{Y}_{j}^{(n)}= & L\left(t_{n}+c_{j} h_{n}\right) Y_{j}^{(n)}+M\left(t_{n}+c_{j} h_{n}\right) Y_{j}^{(n-m)} \\
& +N\left(t_{n}+c_{j} h_{n}\right) \hat{Y}_{j}^{(n-m)}, \quad j=1, \ldots, s, \\
y_{n+1}= & y_{n}+h_{n} \sum_{j=1}^{s} b_{j} \hat{Y}_{j}^{(n)} .
\end{aligned}
$$

Now we present a preliminary result that will be used further on. For the difference equation

$$
\begin{aligned}
u_{n} & =\lambda_{1} u_{n-m}+\lambda_{2} v_{n}+\lambda_{3} v_{n-m}, \\
v_{n+1} & =\lambda_{4} v_{n}+\lambda_{5} u_{n},
\end{aligned}
$$

with $\lambda_{i} \in \mathbb{C}$, we have the asymptotic stability result stated in the following lemma.

LEMma 3.3. The difference equation (3.8)-(3.9) is asymptotically stable if

$$
\left|\lambda_{1}\right|<1, \quad\left|\lambda_{4}\right|<1, \quad\left(\left|\lambda_{2}\right|+\left|\lambda_{3}\right|\right)\left|\lambda_{5}\right|<\left(1-\left|\lambda_{1}\right|\right)\left(1-\left|\lambda_{4}\right|\right) .
$$

Proof. It is easy to see that the characteristic equation of (3.8)-(3.9) is given by

$$
\operatorname{det}\left[\begin{array}{cc}
1-\lambda_{1} z^{-m} & -\lambda_{2}-\lambda_{3} z^{-m} \\
-\lambda_{5} & z-\lambda_{4}
\end{array}\right]=0
$$

which gives

$$
\lambda_{5}\left(\lambda_{2}+\lambda_{3} z^{-m}\right)=z\left(1-\lambda_{4} z^{-1}\right)\left(1-\lambda_{1} z^{-m}\right) .
$$

Suppose $\left|\lambda_{1}\right|<1,\left|\lambda_{4}\right|<1$, and there exists $z \in \mathbb{C}$ satisfying (3.11) with $|z| \geq 1$. Then

$$
\left|\lambda_{5}\right|\left(\left|\lambda_{2}\right|+\left|\lambda_{3}\right|\right) \geq\left(1-\left|\lambda_{4}\right|\right)\left(1-\left|\lambda_{1}\right|\right),
$$

which contradicts the third inequality of (3.10). This completes the proof.

Now we state and prove the main result of this section.

TheOrem 3.4. Let Assumption $\mathcal{A}$ hold and the matrix $A$ be nonsingular. Then the difference equation (3.5-3.7) is asymptotically stable if the underlying Runge-Kutta method is strictly stable at infinity.

Proof. It follows from (3.6) that

$$
\begin{aligned}
L^{-1}\left(t_{n}+c_{i} h_{n}\right) \hat{Y}_{i}^{(n)}= & Y_{i}^{(n)}+L^{-1}\left(t_{n}+c_{i} h_{n}\right) M\left(t_{n}+c_{i} h_{n}\right) Y_{i}^{(n-m)} \\
& +L^{-1}\left(t_{n}+c_{i} h_{n}\right) N\left(t_{n}+c_{i} h_{n}\right) \hat{Y}_{i}^{(n-m)}, \quad i=1, \ldots, s,
\end{aligned}
$$

which in combination with Assumption $\mathcal{A}$ gives

$$
\left\|Y_{i}^{(n)}\right\|_{*} \leq k_{0}\left\|Y_{i}^{(n-m)}\right\|_{*}+C_{0}\left\|\hat{Y}_{i}^{(n)}\right\|_{*}+\hat{C}_{0}\left\|\hat{Y}_{i}^{(n-m)}\right\|_{*} .
$$


On the other hand, (3.5) plus the nonsingularity of $A$ implies

$$
\hat{Y}_{i}^{(n)}=h_{n}^{-1} \sum_{j=1}^{s} D_{i j}\left(Y_{j}^{(n)}-y_{n}\right),
$$

where $D=\left[D_{i j}\right]=A^{-1}$. Substituting (3.13) into (3.7) yields

$$
y_{n+1}=R(\infty) y_{n}+\sum_{i=1}^{s} \sum_{j=1}^{s} b_{i} D_{i j} Y_{j}^{(n)} .
$$

Hence, there exists a constant $C_{1}>0$ such that

$$
\left\|y_{n+1}\right\|_{*} \leq|R(\infty)|\left\|y_{n}\right\|_{*}+C_{1} \sum_{i=1}^{s}\left\|Y_{i}^{(n)}\right\|_{*}
$$

A combination of (3.12) and (3.13) leads to

$$
\begin{aligned}
\sum_{i=1}^{s}\left\|Y_{i}^{(n)}\right\|_{*} \leq & k_{0} \sum_{i=1}^{s}\left\|Y_{i}^{(n-m)}\right\|_{*} \\
& +\sum_{i=1}^{s} \sum_{j=1}^{s}\left|D_{i j}\right|\left(h_{n}^{-1} C_{0}\left\|Y_{j}^{(n)}-y_{n}\right\|_{*}+h_{n-m}^{-1} \hat{C}_{0}\left\|Y_{j}^{(n-m)}-y_{n-m}\right\|_{*}\right)
\end{aligned}
$$

which shows that there exists a constant $\hat{C}_{1}>0$ such that

$$
\begin{aligned}
& \sum_{i=1}^{s}\left\|Y_{i}^{(n)}\right\|_{*} \leq k_{0} \sum_{i=1}^{s}\left\|Y_{i}^{(n-m)}\right\|_{*} \\
& \quad+\hat{C}_{1}\left[h_{n}^{-1}\left(\sum_{j=1}^{s}\left\|Y_{j}^{(n)}\right\|_{*}+\left\|y_{n}\right\|_{*}\right)+h_{n-m}^{-1}\left(\sum_{j=1}^{s}\left\|Y_{j}^{(n-m)}\right\|_{*}+\left\|y_{n-m}\right\|_{*}\right)\right] .
\end{aligned}
$$

Considering $h_{n} \rightarrow \infty$, there exist positive numbers $\mathcal{N}_{0}, k_{1}<1$, and $C_{2}<\left(1-k_{1}\right)(1-$ $|R(\infty)|) /\left(2 C_{1}\right)$ such that for every $n>\mathcal{N}_{0}$,

$$
\sum_{i=1}^{s}\left\|Y_{i}^{(n)}\right\|_{*} \leq k_{1} \sum_{i=1}^{s}\left\|Y_{i}^{(n-m)}\right\|_{*}+C_{2}\left(\left\|y_{n}\right\|_{*}+\left\|y_{n-m}\right\|_{*}\right) .
$$

An application of Lemma 3.3 to (3.15) and (3.17) gives

$$
\lim _{n \rightarrow \infty}\left\|y_{n}\right\|_{*}=0 \quad \text { and } \quad \lim _{n \rightarrow \infty} \sum_{i=1}^{s}\left\|Y_{i}^{(n)}\right\|_{*}=0 .
$$

Considering (3.13), we have

$$
\lim _{n \rightarrow \infty} \sum_{i=1}^{s} h_{n}\left\|\hat{Y}_{i}^{(n)}\right\|_{*}=0
$$

Therefore, the difference equation (3.5-3.7) is asymptotically stable. This completes the proof. 
Remark 3.5. In the case of constant coefficients, it is well known that the condition $\rho\left[L^{-1} M\right]<1$ is equivalent to the condition that there exists a norm $\|\cdot\|_{*}$ such that $\left\|L^{-1} M\right\|_{*}<1$. Therefore, specializing Theorem 3.4 to the case of autonomous equations, the obtained result is in accordance with that by Koto [21]. Here we have given a new approach to the proof which allowed us to study the variable coefficient case.

Remark 3.6. In the proof we only use the fact that $h_{n} \rightarrow \infty$. Hence, our result is also valid for the other grid types proposed in $[2,24]$. In addition, it is easily seen from the proof that, if the condition $\left\|L^{-1}(t) M(t)\right\|_{*} \leq k_{0}<1$ in Assumption $\mathcal{A}$ is replaced by $\lim _{t \rightarrow \infty}\left\|L^{-1}(t) M(t)\right\|_{*} \leq k_{0}<1$, Theorem 3.4 still holds. In the onedimensional case, the latter has been assumed for the stability analysis of $\theta$-methods in [2]. Finally, specializing Theorem 3.4 to the nonneutral case, the induced result is also new.

Next, we show that the assumption of strict stability at infinity is also necessary for the asymptotic stability of the difference equation.

Lemma 3.7. Suppose the matrix $A$ is nonsingular. Then there exists a constant $\mathcal{N}_{1}>0$ such that

$$
|R(z)| \geq|R(\infty)|-2\left|z^{-1}\right|\left|b^{T} A^{-2} e\right|, \quad|z| \geq \mathcal{N}_{1}, z \in \mathbb{C} .
$$

Proof. Considering the nonsingularity of $A$ and the fact

$$
\left(A-z^{-1} I_{s}\right)^{-1}=A^{-1}+z^{-1} A^{-1}\left(A-z^{-1} I_{s}\right)^{-1},
$$

we have

$$
R(z)=R(\infty)-z^{-1} b^{T} A^{-1}\left(A-z^{-1} I_{s}\right)^{-1} e,
$$

which gives

$$
|R(z)| \geq|R(\infty)|-\left|z^{-1}\right|\left|b^{T} A^{-1}\left(A-z^{-1} I_{s}\right)^{-1} e\right| .
$$

Considering

$$
\lim _{z \rightarrow \infty}\left|b^{T} A^{-1}\left(A-z^{-1} I_{s}\right)^{-1} e\right|=\left|b^{T} A^{-2} e\right|,
$$

there exists a constant $\mathcal{N}_{1}$ such that for every $z \in \mathbb{C}$ with $|z| \geq \mathcal{N}_{1}$,

$$
\left|b^{T} A^{-1}\left(A-z^{-1} I_{s}\right)^{-1} e\right| \leq 2\left|b^{T} A^{-2} e\right|,
$$

which, together with (3.19), implies the conclusion.

The application of method (2.4-2.6) to the scalar equation

$$
y^{\prime}(t)=\lambda y(t), \quad \lambda \in \mathbb{R},
$$

leads to the difference equation

$$
y_{n+1}=R\left(h_{n} \lambda\right) y_{n}
$$

THEOREM 3.8. Suppose the matrix $A$ is nonsingular and there exists a constant $\lambda_{0}$ such that the difference equation (3.21) is asymptotically stable for every $\lambda$ satisfying $\lambda \lambda_{0}>\lambda_{0}^{2}$. Then the underlying Runge-Kutta method is strictly stable at infinity. 
Proof. Suppose $|R(\infty)| \geq 1$. By the assumptions of the theorem we can choose $\lambda$ such that

$$
\lambda \lambda_{0}>\lambda_{0}^{2}, \quad\left|h_{i} \lambda\right| \geq \mathcal{N}_{1}, \quad \text { and } \quad\left|h_{i} \lambda\right|>4\left|b^{T} A^{-2} e\right|, \quad i=0,1,
$$

which gives

$$
\left|h_{n} \lambda\right| \geq \mathcal{N}_{1} \quad \text { and } \quad\left|h_{n} \lambda\right|>4\left|b^{T} A^{-2} e\right|, \quad n=0,1,2, \ldots .
$$

Considering Lemma 3.7, we have

$$
\begin{aligned}
\left|R\left(h_{n} \lambda\right)\right| & \geq|R(\infty)|-2\left|h_{n} \lambda\right|^{-1}\left|b^{T} A^{-2} e\right| \\
& \geq|R(\infty)| \exp \left(-4\left|h_{n} \lambda\right|^{-1}\left|b^{T} A^{-2} e\right| /|R(\infty)|\right) \\
& =|R(\infty)| \exp \left(-4 p^{-n+1}\left|h_{1} \lambda\right|^{-1}\left|b^{T} A^{-2} e\right| /|R(\infty)|\right)
\end{aligned}
$$

where we have used the fact that the function $1-x-\exp (-2 x)$ is positive for $x \in$ $(0,1 / 2)$. Therefore,

$$
\begin{aligned}
\prod_{i=1}^{n}\left|R\left(h_{i} \lambda\right)\right| & \geq \prod_{i=1}^{n}|R(\infty)| \exp \left(-4 p^{-i+1}\left|h_{1} \lambda\right|^{-1}\left|b^{T} A^{-2} e\right| /|R(\infty)|\right) \\
& =|R(\infty)|^{n} \exp \left(-\frac{1-p^{-n}}{1-p^{-1}} 4\left|h_{1} \lambda\right|^{-1}\left|b^{T} A^{-2} e\right| /|R(\infty)|\right),
\end{aligned}
$$

which shows that the difference equation (3.21) is not asymptotically stable. This completes the proof.

COROLlary 3.9. Suppose the matrix $A$ is nonsingular and there exists a constant $\lambda_{0}<0$ such that (3.21) is asymptotically stable for every $\lambda \in\left(-\infty, \lambda_{0}\right]$. Then the underlying Runge-Kutta method is strictly stable at infinity.

4. An upper bound of error growth for linear problems. Asymptotic stability implies that the initial error will eventually vanish for sufficiently large time points. From the viewpoint of a practical computation, it is also important to give an upper bound of error growth. This subject was studied in Koto [21], where the nonneutral pantograph equation

$$
\left\{\begin{array}{l}
y^{\prime}(t)=L y(t)+M y(q t), \quad t>0 \\
y(0)=y_{0}
\end{array}\right.
$$

was used as a test problem and algebraically stable methods were considered. In this section we follow Koto's practice and pursue a sharper result for (4.1), which can be regarded as an error equation of a linear problem.

The application of method (2.4-2.6) to (4.1) yields

$$
\begin{aligned}
& Y^{(n)}=\left(e \otimes I_{d}\right) y_{n}+h_{n}\left(A \otimes I_{d}\right) \hat{Y}^{(n)}, \\
& \hat{Y}^{(n)}=\left(I_{s} \otimes L\right) Y^{(n)}+\left(I_{s} \otimes M\right) Y^{(n-m)}, \\
& y_{n+1}=y_{n}+h_{n}\left(b^{T} \otimes I_{d}\right) \hat{Y}^{(n)},
\end{aligned}
$$

where $\otimes$ denotes the Kronecker product and

$$
Y^{(n)}=\left(Y_{1}^{(n)^{T}}, Y_{2}^{(n)^{T}}, \ldots, Y_{s}^{(n)^{T}}\right)^{T}, \quad \hat{Y}^{(n)}=\left(\hat{Y}_{1}^{(n)^{T}}, \hat{Y}_{2}^{(n)^{T}}, \ldots, \hat{Y}_{s}^{(n)^{T}}\right)^{T}
$$


The following notation is a generalization of that in [21]:

$$
\mathcal{H}(\sigma)=-\left[\begin{array}{cc}
L^{*} G+G L+E & G M \\
M^{*} G & -\sigma E
\end{array}\right]
$$

where $G$ and $E$ are Hermitian positive definite matrices and the superscript $*$ stands for the Hermitian adjoint. It is also seen that $\mathcal{H}\left(\sigma_{2}\right)$ is necessarily nonnegative definite if $\mathcal{H}\left(\sigma_{1}\right)$ is nonnegative definite and $\sigma_{2}>\sigma_{1}$. In the scalar case, if the complex numbers $L, M$ satisfy

$$
\sqrt{\sigma}(\operatorname{Re} L)+|M|<0
$$

then $\mathcal{H}(\sigma)$ is nonnegative definite.

Throughout this section, we assume that the notations are the same as those in section 3 .

Lemma 4.1. Suppose that the method $(A, b, c)$ is algebraically stable and there exist a constant $\sigma$ and matrices $G$ and $E$ such that $\mathcal{H}(\sigma)$ is nonnegative definite. Then the following inequality holds true:

$$
y_{n+1}^{*} G y_{n+1} \leq y_{n}^{*} G y_{n}-h_{n} Y^{(n)^{*}}(B \otimes E) Y^{(n)}+\sigma h_{n} Y^{(n-m)^{*}}(B \otimes E) Y^{(n-m)} .
$$

Proof. As in Burrage and Butcher [10], where it is proved that algebraic stability implies B-stability, we can obtain

$$
\begin{aligned}
& y_{n+1}^{*} G y_{n+1}-y_{n}^{*} G y_{n}-h_{n} Y^{(n)^{*}}(B \otimes G) \hat{Y}^{(n)}-h_{n} \hat{Y}^{(n)^{*}}(B \otimes G) Y^{(n)} \\
& \quad=-h_{n}^{2} \hat{Y}^{(n)^{*}}(\mathcal{M} \otimes G) \hat{Y}^{(n)} .
\end{aligned}
$$

By using the algebraic stability of the method, we have

$$
\begin{aligned}
y_{n+1}^{*} G y_{n+1} \leq & y_{n}^{*} G y_{n}+h_{n} Y^{(n)^{*}}(B \otimes G) \hat{Y}^{(n)}+h_{n} \hat{Y}^{(n)^{*}}(B \otimes G) Y^{(n)} \\
= & y_{n}^{*} G y_{n}-h_{n} Y^{(n)^{*}}(B \otimes E) Y^{(n)}+\sigma h_{n} Y^{(n-m)^{*}}(B \otimes E) Y^{(n-m)} \\
& -h_{n} \sum_{i=1}^{s} b_{i}\left(Y_{i}^{(n)^{*}}, Y_{i}^{(n-m)^{*}}\right) \mathcal{H}(\sigma)\left(Y_{i}^{(n)^{T}}, Y_{i}^{(n-m)^{T}}\right)^{T}
\end{aligned}
$$

which by the nonnegative definiteness of $\mathcal{H}(\sigma)$ gives (4.6).

THEOREM 4.2. Suppose that the method $(A, b, c)$ is algebraically stable and there exist matrices $G$ and $E$ such that $\mathcal{H}(q)$ is nonnegative definite. Then we have that for every $n \geq m$,

$$
y_{n+1}^{*} G y_{n+1}+\sum_{i=n-m+1}^{n} h_{i} Y^{(i)^{*}}(B \otimes E) Y^{(i)} \leq y_{n}^{*} G y_{n}+\sum_{i=n-m}^{n-1} h_{i} Y^{(i)^{*}}(B \otimes E) Y^{(i)} .
$$

Proof. Inequality (4.7) immediately follows from Lemma 4.1 and the fact $q h_{n}=$ $h_{n-m}$.

Remark 4.3. By the same argument as in section 5 of Koto [21], it is seen that the functional

$$
V(y(t))=y(t)^{*} G y(t)+\int_{q t}^{t} y(x)^{*} E y(x) d x
$$


is a Liapunov functional for equation $(4.1)$ if $\mathcal{H}(q)$ is nonnegative definite. Inequality (4.7) can be regarded as a discrete analogue.

Remark 4.4. The proof of Lemma 4.1 is closely related to its counterpart in [21]. There, (4.1) was studied through an investigation of the corresponding constant delay system transformed by a change of independent variable.

In the following, we derive a result which can be applied to the more general case where $\mathcal{H}(\sigma)$ is nonnegative definite for some $\sigma \in[q, 1)$. Except for certain special statements, the following results remain valid for $\sigma \geq 1$ although they may not result in stability when $\sigma \geq 1$.

Lemma 4.5. Suppose that the method $(A, b, c)$ is algebraically stable and there exist a constant $\sigma \geq q$ and matrices $G$ and $E$ such that $\mathcal{H}(\sigma)$ is nonnegative definite. Then we have that for every $k \geq 0$,

$$
\sum_{i=1}^{m} p^{-i} Y^{((k+2) m-i)^{*}}(B \otimes E) Y^{((k+2) m-i)} \leq q^{2} \sigma^{k} \delta
$$

where

$$
\delta=p h_{1}^{-1} y_{m}^{*} G y_{m}+q^{-1} \sigma \sum_{i=0}^{m-1} p^{i} Y^{(i)^{*}}(B \otimes E) Y^{(i)} .
$$

Proof. It follows from Lemma 4.1 that

$$
\begin{aligned}
y_{n+1}^{*} G y_{n+1} \leq & y_{n}^{*} G y_{n}-h_{1} p^{n-1} Y^{(n)^{*}}(B \otimes E) Y^{(n)} \\
& +\sigma q^{-1} h_{1} p^{n-m-1} Y^{(n-m)^{*}}(B \otimes E) Y^{(n-m)} .
\end{aligned}
$$

By induction, one arrives at

$$
\begin{aligned}
y_{n+1}^{*} G y_{n+1} \leq & y_{m}^{*} G y_{m}-h_{1} \sum_{i=n-m+1}^{n} p^{i-1} Y^{(i)^{*}}(B \otimes E) Y^{(i)} \\
& +\left(-1+\sigma q^{-1}\right) h_{1} \sum_{i=m}^{n-m} p^{i-1} Y^{(i)^{*}}(B \otimes E) Y^{(i)} \\
& +\sigma q^{-1} h_{1} \sum_{i=0}^{m-1} p^{i-1} Y^{(i)^{*}}(B \otimes E) Y^{(i)} .
\end{aligned}
$$

Therefore,

$$
\sum_{i=n-m+1}^{n} p^{i} Y^{(i)^{*}}(B \otimes E) Y^{(i)} \leq \delta+\left(-1+\sigma q^{-1}\right) \sum_{i=m}^{n-m} p^{i} Y^{(i)^{*}}(B \otimes E) Y^{(i)} .
$$

Let $n=(k+2) m-1$ for $k \geq 0$. We have

$$
\begin{aligned}
p^{(k+2) m} & \sum_{i=1}^{m} p^{-i} Y^{((k+2) m-i)^{*}}(B \otimes E) Y^{((k+2) m-i)} \\
\leq & \delta+\left(-1+\sigma q^{-1}\right) \sum_{l=0}^{k-1} p^{(l+2) m} \sum_{i=1}^{m} p^{-i} Y^{((l+2) m-i)^{*}}(B \otimes E) Y^{((l+2) m-i)},
\end{aligned}
$$


which gives

$$
\begin{aligned}
& \sum_{i=1}^{m} p^{-i} Y^{((k+2) m-i)^{*}}(B \otimes E) Y^{((k+2) m-i)} \\
& \quad \leq q^{k+2} \delta+\left(-1+\sigma q^{-1}\right) \sum_{l=1}^{k} q^{l} \sum_{i=1}^{m} p^{-i} Y^{((k+2-l) m-i)^{*}}(B \otimes E) Y^{((k+2-l) m-i)} .
\end{aligned}
$$

We prove (4.8) by induction. When $k=0,(4.8)$ follows directly from the above inequality. Now we assume that (4.8) holds for every $k<j$ and show that it then also holds for $k=j$. It follows from the above inequality that

$$
\begin{aligned}
& \sum_{i=1}^{m} p^{-i} Y^{((j+2) m-i)^{*}}(B \otimes E) Y^{((j+2) m-i)} \\
& \quad \leq q^{j+2} \delta+\left(-1+\sigma q^{-1}\right) \sum_{l=1}^{j} q^{l} \sum_{i=1}^{m} p^{-i} Y^{((j+2-l) m-i)^{*}}(B \otimes E) Y^{((j+2-l) m-i)} \\
& \quad \leq q^{j+2} \delta+\left(-1+\sigma q^{-1}\right) \sum_{l=1}^{j} q^{l+2} \sigma^{j-l} \delta \\
& \quad=q^{2} \sigma^{j} \delta .
\end{aligned}
$$

Therefore, (4.8) holds for every $k \geq 0$. This completes the proof.

COROLLARY 4.6. Under the assumptions of Lemma 4.5, (4.8) implies that,

$$
Y^{(n)^{*}}(B \otimes E) Y^{(n)} \leq \sigma^{n / m} \delta, \quad \text { for every } n \geq m .
$$

Proof. It follows from (4.8) that for every $k \geq 0, i \in\{1, \ldots, m\}$,

$$
Y^{((k+2) m-i)^{*}}(B \otimes E) Y^{((k+2) m-i)} \leq p^{i} q^{2} \sigma^{k} \delta=q^{2-i / m} \sigma^{k} \delta \leq \sigma^{k+2-i / m} \delta,
$$

which gives (4.10).

THEOREM 4.7. Suppose that the method $(A, b, c)$ with a nonsingular matrix $A$ is algebraically stable, $b_{i}>0$ for all $i$ and there exist a constant $\sigma \geq q$ and matrices $G$ and $E$ such that $\mathcal{H}(\sigma)$ is nonnegative definite. Then there exists a constant $\bar{C}$ such that for every $n \geq m$,

$$
\left\|y_{n+1}\right\|_{*} \leq|R(\infty)|^{n+1-m}\left\|y_{m}\right\|_{*}+\bar{C} \sigma^{1 / 2} \delta^{1 / 2} \psi_{n+1-m}\left(|R(\infty)|, \sigma^{1 / 2 m}\right),
$$

where $\|\cdot\|_{*}$ denotes a norm on $\mathbb{C}^{d}$, and

$$
\psi_{n}(x, y)= \begin{cases}\frac{x^{n}-y^{n}}{x-y}, & x \neq y, \\ n x^{n-1}, & x=y .\end{cases}
$$

Proof. Since $b_{i}>0$ for all $i$, it follows from Corollary 4.6 that there exists a constant $\bar{C}_{1}$ such that for every $n \geq m$

$$
\sum_{j=1}^{s}\left\|Y_{j}^{(n)}\right\|_{*} \leq \bar{C}_{1} \sigma^{n / 2 m} \delta^{1 / 2} .
$$


Considering (3.14) and the assumptions from the statement of the theorem, there exists a constant $\bar{C}_{2}$ such that

$$
\left\|y_{n+1}\right\|_{*} \leq|R(\infty)|\left\|y_{n}\right\|_{*}+\bar{C}_{2} \sum_{j=1}^{s}\left\|Y_{j}^{(n)}\right\|_{*} .
$$

By induction, we have

$$
\begin{aligned}
\left\|y_{n+1}\right\|_{*} & \leq|R(\infty)|\left\|y_{n}\right\|_{*}+\bar{C}_{1} \bar{C}_{2} \sigma^{n / 2 m} \delta^{1 / 2} \\
& \leq|R(\infty)|^{n+1-m}\left\|y_{m}\right\|_{*}+\bar{C}_{1} \bar{C}_{2} \sigma^{1 / 2} \delta^{1 / 2} \sum_{i=0}^{n-m}|R(\infty)|^{i} \sigma^{(n-m-i) / 2 m} .
\end{aligned}
$$

This implies inequality (4.12).

Using the fact that $R(\infty)=0$ for the Radau IA, Radau IIA, and Lobatto IIIC methods, and the fact that $|R(\infty)|=1$ for the Gauss methods, we can state the following corollaries.

COROLlary 4.8. Suppose that there exist a constant $\sigma \geq q$ and matrices $G$ and $E$ such that $\mathcal{H}(\sigma)$ is nonnegative definite. Then for any Radau IA, Radau IIA, or Lobatto IIIC method, there exists a constant $\bar{C}$ such that for every $n \geq m$,

$$
\left\|y_{n+1}\right\|_{*} \leq \bar{C} \sigma^{n / 2 m} \delta^{1 / 2}
$$

Corollary 4.9. Suppose that there exist a constant $\sigma \in[q, 1)$ and matrices $G$ and $E$ such that $\mathcal{H}(\sigma)$ is nonnegative definite. Then for any Gauss method, there exists a constant $\bar{C}$ such that for every $n \geq m$,

$$
\left\|y_{n+1}\right\|_{*} \leq\left\|y_{m}\right\|_{*}+\frac{\bar{C} \sigma^{1 / 2} \delta^{1 / 2}}{1-\sigma^{1 / 2 m}}
$$

Remark 4.10. The Assumption (L) in Koto [21] is equivalent to the condition that there exist matrices $G$ and $E$ such that $\mathcal{H}(q)$ is nonnegative definite. Our result can be applied to the more general case $\sigma \geq q$.

5. Nonlinear stability. In this section, we derive conditions which guarantee the asymptotic stability of the numerical solution of nonlinear equations. First, we recall a result on the asymptotic stability of the analytical solution. Consider a system defined by the same function $f$ as in (2.1) but with a different initial value,

$$
\left\{\begin{array}{l}
z^{\prime}(t)=f\left(t, z(t), z(q t), z^{\prime}(q t)\right), \quad t>0 \\
z(0)=z_{0}
\end{array}\right.
$$

Let $\langle\cdot, \cdot\rangle$ be an inner product on $\mathbb{C}^{d}$, let $\|\cdot\|$ be the corresponding norm, and let the function $f$ satisfy the conditions

$$
\begin{array}{r}
\operatorname{Re}\left\langle u_{1}-u_{2}, f\left(t, u_{1}, v, \nu\right)-f\left(t, u_{2}, v, \nu\right)\right\rangle \leq \alpha(t)\left\|u_{1}-u_{2}\right\|^{2}, \\
\text { for } t>0, u_{1}, u_{2}, v, \nu \in \mathbb{C}^{d}, \\
\left\|f\left(t, u, v_{1}, \nu_{1}\right)-f\left(t, u, v_{2}, \nu_{2}\right)\right\| \leq \beta(t)\left\|v_{1}-v_{2}\right\|+\gamma(t)\left\|\nu_{1}-\nu_{2}\right\|, \\
\text { for } t>0, u, v_{1}, v_{2}, \nu_{1}, \nu_{2} \in \mathbb{C}^{d},
\end{array}
$$

where $\alpha(t), \beta(t)$, and $\gamma(t)$ are continuous functions. An application of Theorem 2.1 in Zennaro [27] (see also [5]) gives the following proposition. 
Proposition 5.1. Suppose $\gamma(t)=0$ and the functions $\alpha(t)$ and $\beta(t)$ satisfy

$$
\alpha(t) \leq \alpha_{0}<0, \quad t>0,
$$

and, for some nonnegative real number $k_{0}<1$,

$$
k_{0} \alpha(t)+\beta(t) \leq 0, \quad t>0 .
$$

Then, for the solutions $y(t)$ and $z(t)$ of (2.1) and (5.1), it holds that

$$
\lim _{t \rightarrow \infty}\|y(t)-z(t)\|=0 .
$$

Remark 5.2. In the literature, we have not found any stability results on general nonlinear neutral equations. Some results on equations of special form, such as separable systems and equations of Hale's form, can be found in $[3,19,26]$. We do not give the details of those results because they cannot directly be applied to equations of the form (2.1). Here, we will only use conditions (5.4) and (5.5) plus the boundedness of $\gamma(t)$ to analyze the asymptotic stability of numerical methods for nonlinear neutral equations of the form (2.1). Stability results for nonneutral equations are given in [5] (Theorem 9.7.1).

The Runge-Kutta method $(A, b, c)$ applied to problem (5.1) leads to the following process:

$$
\begin{aligned}
Z_{i}^{(n)} & =z_{n}+h_{n} \sum_{j=1}^{s} a_{i j} \hat{Z}_{j}^{(n)}, \quad i=1, \ldots, s, \\
\hat{Z}_{j}^{(n)} & =f\left(t_{n}+c_{j} h_{n}, Z_{j}^{(n)}, Z_{j}^{(n-m)}, \hat{Z}_{j}^{(n-m)}\right), \quad j=1, \ldots, s, \\
z_{n+1} & =z_{n}+h_{n} \sum_{j=1}^{s} b_{j} \hat{Z}_{j}^{(n)} .
\end{aligned}
$$

Let

$$
w_{n}=y_{n}-z_{n}, \quad W_{j}^{(n)}=Y_{j}^{(n)}-Z_{j}^{(n)}, \quad j=1, \ldots, s .
$$

It follows from $(2.4-2.6)$ and $(5.7-5.9)$ that

$$
\begin{aligned}
& W_{i}^{(n)}=w_{n}+h_{n} \sum_{j=1}^{s} a_{i j}\left(\hat{Y}_{j}^{(n)}-\hat{Z}_{j}^{(n)}\right), \quad i=1, \ldots, s, \\
& w_{n+1}=w_{n}+h_{n} \sum_{j=1}^{s} b_{j}\left(\hat{Y}_{j}^{(n)}-\hat{Z}_{j}^{(n)}\right) .
\end{aligned}
$$

Now we are in the position to state and prove the main result of this section.

THEOREM 5.3. Suppose that the method $(A, b, c)$ with a nonsingular matrix $A$ is strictly stable at infinity and that there exist positive constants $C_{3}, C_{4}$, and $k_{2}$ such that

$$
0<-\alpha^{-1}(t) \leq C_{3}, \quad\left|\alpha^{-1}(t) \gamma(t)\right| \leq C_{4}, \quad\left|\alpha^{-1}(t) \beta(t)\right| \leq k_{2}<1 .
$$

Then, the following results hold:

$$
\begin{gathered}
\lim _{n \rightarrow \infty}\left\|w_{n}\right\|=0, \quad \lim _{n \rightarrow \infty} \sum_{j=1}^{s}\left\|W_{j}^{(n)}\right\|=0, \\
\lim _{n \rightarrow \infty} h_{n} \sum_{j=1}^{s}\left\|\hat{Y}_{j}^{(n)}-\hat{Z}_{j}^{(n)}\right\|=0 .
\end{gathered}
$$


Proof. From (5.10) and the nonsingularity of $A$ it follows that

$$
\hat{Y}_{i}^{(n)}-\hat{Z}_{i}^{(n)}=h_{n}^{-1} \sum_{j=1}^{s} D_{i j}\left(W_{j}^{(n)}-w_{n}\right), \quad i=1, \ldots, s,
$$

where $D=\left[D_{i j}\right]=A^{-1}$. Substituting (5.15) into (5.11) yields

$$
w_{n+1}=R(\infty) w_{n}+\sum_{i=1}^{s} \sum_{j=1}^{s} b_{i} D_{i j} W_{j}^{(n)} .
$$

Hence, there exists a constant $C_{5}>0$ such that

$$
\left\|w_{n+1}\right\| \leq|R(\infty)|\left\|w_{n}\right\|+C_{5} \sum_{i=1}^{s}\left\|W_{i}^{(n)}\right\| .
$$

On the other hand, conditions (5.2) and (5.3) imply that $\operatorname{Re}\left\langle W_{j}^{(n)}, \hat{Y}_{j}^{(n)}-\hat{Z}_{j}^{(n)}\right\rangle$ can be rewritten and bounded as follows:

$$
\begin{aligned}
& \operatorname{Re}\left\langle W_{j}^{(n)}, f\left(t_{n}+c_{j} h_{n}, Y_{j}^{(n)}, Y_{j}^{(n-m)}, \hat{Y}_{j}^{(n-m)}\right)-f\left(t_{n}+c_{j} h_{n}, Z_{j}^{(n)}, Y_{j}^{(n-m)}, \hat{Y}_{j}^{(n-m)}\right)\right\rangle \\
& \quad+\operatorname{Re}\left\langle W_{j}^{(n)}, f\left(t_{n}+c_{j} h_{n}, Z_{j}^{(n)}, Y_{j}^{(n-m)}, \hat{Y}_{j}^{(n-m)}\right)-f\left(t_{n}+c_{j} h_{n}, Z_{j}^{(n)}, Z_{j}^{(n-m)}, \hat{Z}_{j}^{(n-m)}\right)\right\rangle \\
& \quad \leq \alpha\left(t_{n}+c_{j} h_{n}\right)\left\|W_{j}^{(n)}\right\|^{2}+\beta\left(t_{n}+c_{j} h_{n}\right)\left\|W_{j}^{(n)}\right\|\left\|W_{j}^{(n-m)}\right\| \\
& \quad+\gamma\left(t_{n}+c_{j} h_{n}\right)\left\|W_{j}^{(n)}\right\|\left\|\hat{Y}_{j}^{(n-m)}-\hat{Z}_{j}^{(n-m)}\right\| .
\end{aligned}
$$

Considering the inequality

$$
\operatorname{Re}\left\langle W_{j}^{(n)}, \hat{Y}_{j}^{(n)}-\hat{Z}_{j}^{(n)}\right\rangle \geq-\left\|W_{j}^{(n)}\right\|\left\|\hat{Y}_{j}^{(n)}-\hat{Z}_{j}^{(n)}\right\|,
$$

we have that

$$
\left\|W_{j}^{(n)}\right\| \leq k_{2}\left\|W_{j}^{(n-m)}\right\|+C_{4}\left\|\hat{Y}_{j}^{(n-m)}-\hat{Z}_{j}^{(n-m)}\right\|+C_{3}\left\|\hat{Y}_{j}^{(n)}-\hat{Z}_{j}^{(n)}\right\|,
$$

where we have used Assumption (5.12). Using (5.15), we further obtain

$$
\begin{aligned}
\sum_{i=1}^{s}\left\|W_{i}^{(n)}\right\| \leq & k_{2} \sum_{i=1}^{s}\left\|W_{i}^{(n-m)}\right\|+h_{n-m}^{-1} C_{4} \sum_{i=1}^{s} \sum_{j=1}^{s}\left|D_{i j}\right|\left\|W_{j}^{(n-m)}-w_{n-m}\right\| \\
& +h_{n}^{-1} C_{3} \sum_{i=1}^{s} \sum_{j=1}^{s}\left|D_{i j}\right|\left\|W_{j}^{(n)}-w_{n}\right\| .
\end{aligned}
$$

Considering $h_{n} \rightarrow \infty$, there exist positive numbers $\mathcal{N}_{2}, k_{3}<1$ and $C_{6}<\left(1-k_{3}\right)(1-$ $|R(\infty)|) /\left(2 C_{5}\right)$ such that for every $n>\mathcal{N}_{2}$,

$$
\sum_{i=1}^{s}\left\|W_{i}^{(n)}\right\| \leq k_{3} \sum_{i=1}^{s}\left\|W_{i}^{(n-m)}\right\|+C_{6}\left(\left\|w_{n}\right\|+\left\|w_{n-m}\right\|\right) .
$$

An application of Lemma 3.3 to (5.17) and (5.20) gives (5.13). Then (5.14) follows from (5.15). This completes the proof.

Remark 5.4. In the proof, we only use $h_{n} \rightarrow \infty$. Hence, the result is also valid for the other grid types proposed in $[2,24]$. From Corollary 3.9 we can see that the assumption of strict stability at infinity is also necessary to the result. 
Remark 5.5. Theorem 5.3 is different from the asymptotic stability result obtained by Zhang and Sun [28] (see Theorem 6.1 of this paper). Therefore, specializing Theorem 5.3 to the case of nonneutral equations, our result is also new. In addition, our proof is completely different from that in [28].

Remark 5.6. It should be pointed out that Theorem 5.3 cannot cover the asymptotic stability results of section 3. In fact, specializing the Assumption (5.12) in Theorem 5.3 to the case of (3.1), the induced assumptions are stronger than Assumption $\mathcal{A}$.

6. An upper bound of error growth for nonlinear problems. In this section, we investigate error growth bounds of numerical methods for nonneutral, nonlinear problems of the form

$$
\left\{\begin{array}{l}
y^{\prime}(t)=f(t, y(t), y(q t)), \quad t>0 \\
y(0)=y_{0}
\end{array}\right.
$$

where the function $f$ satisfies the conditions

$$
\begin{gathered}
\operatorname{Re}\left\langle u_{1}-u_{2}, f\left(t, u_{1}, v\right)-f\left(t, u_{2}, v\right)\right\rangle \leq \alpha\left\|u_{1}-u_{2}\right\|^{2}, \quad t>0, u_{1}, u_{2}, v \in \mathbb{C}^{d} \\
\left\|f\left(t, u, v_{1}\right)-f\left(t, u, v_{2}\right)\right\| \leq \beta\left\|v_{1}-v_{2}\right\|, \quad t>0, u, v_{1}, v_{2} \in \mathbb{C}^{d}
\end{gathered}
$$

where $\alpha$ and $\beta$ are constants. Throughout this section, we assume that the other notations are the same as those in section 5 .

For problems (6.1-6.3), Zhang and Sun (cf. [28]) have considered a stepsize strategy where every interval $\left(q^{-i} h, q^{-i-1} h\right]$ is divided into $m$ subintervals of the same size, and obtained the following global and asymptotic stability results for $(k, l)-$ algebraically stable Runge-Kutta methods. Here, a method $(A, b, c)$ is said to be $(k, l)$-algebraically stable if there exists a nonnegative diagonal matrix $D$ such that the matrix

$$
\left[\begin{array}{cc}
k-1-2 l e^{T} D e & e^{T} D-b^{T}-2 l e^{T} D A \\
D e-b-2 l A^{T} D e & D A+A^{T} D-b b^{T}-2 l A^{T} D A
\end{array}\right]
$$

is nonnegative definite (cf. [11]).

Theorem 6.1 (see [28]). Suppose that the method $(A, b, c)$ is $(k, l)$-algebraically stable for a nonnegative diagonal matrix $D=\operatorname{diag}\left(d_{1}, d_{2}, \ldots, d_{s}\right)$, where $0<k \leq 1$ and the following condition holds:

$$
q \alpha+\beta \leq 0, \quad(1-q)(q \alpha+\beta) h \leq m q^{2} l,
$$

then

$$
\left\|w_{n+1}\right\| \leq\left[1+\sqrt{\left(q^{-1}-1\right) \beta h}\right] \max \left\{\left\|w_{m}\right\|, \sum_{j=1}^{s}\left(\sqrt{b_{j}} \max _{-m \leq i \leq-1}\left\|W_{j}^{(m+i)}\right\|\right)\right\} .
$$

If it is further assumed that $k<1$, then

$$
\lim _{n \rightarrow \infty}\left\|y_{n}-z_{n}\right\|=0 .
$$

Here we obtain the following results. 
TheOREm 6.2. Suppose that the method $(A, b, c)$ is algebraically stable and that the following condition holds:

$$
\alpha+q^{-1 / 2} \beta \leq 0
$$

Then we have that for every $n \geq 2 m-1$,

$$
\begin{gathered}
\left\|w_{n+1}\right\|^{2}-h_{1}\left(2 \alpha+q^{-1 / 2} \beta\right) \sum_{i=n-m+1}^{n} \sum_{j=1}^{s} b_{j} p^{i-1}\left\|W_{j}^{(i)}\right\|^{2} \\
\leq\left\|w_{m}\right\|^{2}+h_{1} q^{-1 / 2} \beta \sum_{i=0}^{m-1} \sum_{j=1}^{s} b_{j} p^{i-1}\left\|W_{j}^{(i)}\right\|^{2}
\end{gathered}
$$

Proof. It is known (see [10]) that

$$
\begin{gathered}
\left\|w_{n+1}\right\|^{2}-\left\|w_{n}\right\|^{2}-2 h_{n} \sum_{j=1}^{s} b_{j} \operatorname{Re}\left\langle W_{j}^{(n)}, \hat{Y}_{j}^{(n)}-\hat{Z}_{j}^{(n)}\right\rangle \\
=-h_{n}^{2} \sum_{i=1}^{s} \sum_{j=1}^{s} \mathcal{M}_{i j}\left\langle\hat{Y}_{i}^{(n)}-\hat{Z}_{i}^{(n)}, \hat{Y}_{j}^{(n)}-\hat{Z}_{j}^{(n)}\right\rangle .
\end{gathered}
$$

By means of the algebraic stability of the method and by (6.2) and (6.3), we have

$$
\begin{aligned}
&\left\|w_{n+1}\right\|^{2} \leq\left\|w_{n}\right\|^{2}+2 h_{n} \sum_{j=1}^{s} b_{j} \operatorname{Re}\left\langle W_{j}^{(n)}, \hat{Y}_{j}^{(n)}-\hat{Z}_{j}^{(n)}\right\rangle \\
&=\left\|w_{n}\right\|^{2}+2 h_{n} \sum_{j=1}^{s} b_{j} \operatorname{Re}\left\langle W_{j}^{(n)}, f\left(t_{n}+c_{j} h_{n}, Y_{j}^{(n)}, Y_{j}^{(n-m)}\right)\right. \\
&\left.\quad-f\left(t_{n}+c_{j} h_{n}, Z_{j}^{(n)}, Y_{j}^{(n-m)}\right)\right\rangle \\
&+2 h_{n} \sum_{j=1}^{s} b_{j} \operatorname{Re}\left\langle W_{j}^{(n)}, f\left(t_{n}+c_{j} h_{n}, Z_{j}^{(n)}, Y_{j}^{(n-m)}\right)\right. \\
&\left.\quad-f\left(t_{n}+c_{j} h_{n}, Z_{j}^{(n)}, Z_{j}^{(n-m)}\right)\right\rangle \\
& \leq\left\|w_{n}\right\|^{2}+h_{n} \sum_{j=1}^{s} b_{j}\left[\left(2 \alpha+q^{-1 / 2} \beta\right)\left\|W_{j}^{(n)}\right\|^{2}+q^{1 / 2} \beta\left\|W_{j}^{(n-m)}\right\|^{2}\right],
\end{aligned}
$$

where we have used

$$
2\left\|W_{j}^{(n)}\right\|\left\|W_{j}^{(n-m)}\right\| \leq q^{-1 / 2}\left\|W_{j}^{(n)}\right\|^{2}+q^{1 / 2}\left\|W_{j}^{(n-m)}\right\|^{2} .
$$

By induction, one arrives at

$$
\begin{aligned}
\left\|w_{n+1}\right\|^{2} \leq\left\|w_{m}\right\|^{2}+h_{1} \sum_{j=1}^{s} b_{j}[ & \sum_{i=n-m+1}^{n} p^{i-1}\left(2 \alpha+q^{-1 / 2} \beta\right)\left\|W_{j}^{(i)}\right\|^{2} \\
& +2 \sum_{i=m}^{n-m} p^{i-1}\left(\alpha+q^{-1 / 2} \beta\right)\left\|W_{j}^{(i)}\right\|^{2} \\
& \left.+\sum_{i=0}^{m-1} p^{i-1} q^{-1 / 2} \beta\left\|W_{j}^{(i)}\right\|^{2}\right]
\end{aligned}
$$


which, combined with (6.6), gives (6.7). This completes the proof.

COROLlary 6.3. Under the assumptions of Theorem 6.2, we have that for every $n \geq 2 m-1$,

$$
\left\|w_{n+1}\right\|^{2} \leq\left\|w_{m}\right\|^{2}+h p^{-1} q^{-1 / 2}(1-q) \beta \max _{\substack{0 \leq i \leq m-1 \\ 1 \leq j \leq s}}\left\|W_{j}^{(i)}\right\|^{2} .
$$

Proof. The conclusion follows from the fact that

$$
\sum_{i=0}^{m-1} p^{i}\left\|W_{j}^{i}\right\|^{2} \leq \frac{p^{m}-1}{p-1} \max _{0 \leq i \leq m-1}\left\|W_{j}^{(i)}\right\|^{2} .
$$

Remark 6.4. The above proof procedure is closely related to its counterpart in the case of a constant delay (cf. [15]) and in the case of a proportional delay (cf. [28]). Compared to Theorem 6.1 in the case of algebraically stable methods, our result is slightly sharper because Assumption (6.4) is stronger than (6.6). by

Remark 6.5. In the case of time-dependent Lipschitz constants, if (6.6) is replaced

$$
2 \alpha(t)+q^{-1 / 2}(\beta(t)+\beta(t / q)) \leq 0,
$$

we can similarly obtain that for every $n \geq 2 m-1$,

$$
\begin{gathered}
\left\|w_{n+1}\right\|^{2}-h_{1} \sum_{i=n-m+1}^{n} \sum_{j=1}^{s} b_{j} p^{i-1}\left(2 \alpha\left(t_{i}+c_{j} h_{i}\right)+q^{-1 / 2} \beta\left(t_{i}+c_{j} h_{i}\right)\right)\left\|W_{j}^{(i)}\right\|^{2} \\
\leq\left\|w_{m}\right\|^{2}+h p^{-1} q^{-1 / 2}(1-q) \max _{\substack{0 \leq i \leq m-1 \\
1 \leq j \leq s}} \beta\left(t_{i+m}+c_{j} h_{i+m}\right)\left\|W_{j}^{(i)}\right\|^{2} .
\end{gathered}
$$

Next, we derive some results which can be applied to the more general case $\alpha+\beta<0$. We define the following two constants

$$
\begin{aligned}
& r=\left\{\begin{array}{lr}
0, & \text { when } 2 \alpha+\left(1+q^{-1}\right) \beta \leq 0, \\
\frac{2 \alpha+\left(1+q^{-1}\right) \beta}{-(2 \alpha+\beta)}, & \text { when } 2 \alpha+\left(1+q^{-1}\right) \beta>0,
\end{array}\right. \\
& \Delta=\frac{\left\|w_{m}\right\|^{2} p / h_{1}+q^{-1} \beta \sum_{j=1}^{s} b_{j} \sum_{i=0}^{m-1} p^{i}\left\|W_{j}^{(i)}\right\|^{2}}{-(2 \alpha+\beta)} .
\end{aligned}
$$

TheOrem 6.6. Suppose that the method $(A, b, c)$ is algebraically stable and that the following condition holds:

$$
\alpha+\beta<0 .
$$

Then we have that for every $k \geq 0$,

$$
\sum_{j=1}^{s} b_{j} \sum_{i=1}^{m} p^{-i}\left\|W_{j}^{((k+2) m-i)}\right\|^{2} \leq q^{2}(q+q r)^{k} \Delta .
$$

Proof. If we replace (6.8) by the inequality

$$
2\left\|W_{j}^{(n)}\right\|\left\|W_{j}^{(n-m)}\right\| \leq\left\|W_{j}^{(n)}\right\|^{2}+\left\|W_{j}^{(n-m)}\right\|^{2},
$$


we obtain

$$
\left\|w_{n+1}\right\|^{2} \leq\left\|w_{n}\right\|^{2}+h_{n} \sum_{j=1}^{s} b_{j}\left[(2 \alpha+\beta)\left\|W_{j}^{(n)}\right\|^{2}+\beta\left\|W_{j}^{(n-m)}\right\|^{2}\right] .
$$

By induction, one arrives at

$$
\begin{array}{r}
\left\|w_{n+1}\right\|^{2} \leq\left\|w_{m}\right\|^{2}+h_{1} p^{-1} \sum_{j=1}^{s} b_{j}\left[\sum_{i=n-m+1}^{n} p^{i}(2 \alpha+\beta)\left\|W_{j}^{(i)}\right\|^{2}\right. \\
\left.+\sum_{i=m}^{n-m} p^{i}\left(2 \alpha+\left(1+q^{-1}\right) \beta\right)\left\|W_{j}^{(i)}\right\|^{2}+\sum_{i=0}^{m-1} p^{i} q^{-1} \beta\left\|W_{j}^{(i)}\right\|^{2}\right] .
\end{array}
$$

Therefore,

$$
\sum_{j=1}^{s} b_{j} \sum_{i=n-m+1}^{n} p^{i}\left\|W_{j}^{(i)}\right\|^{2} \leq r \sum_{j=1}^{s} b_{j} \sum_{i=m}^{n-m} p^{i}\left\|W_{j}^{(i)}\right\|^{2}+\Delta .
$$

Let $n=(k+2) m-1$ for $k \geq 0$. We then have

$$
\sum_{j=1}^{s} b_{j} \sum_{i=1}^{m} p^{-i}\left\|W_{j}^{((k+2) m-i)}\right\|^{2} \leq r \sum_{l=1}^{k} q^{l} \sum_{j=1}^{s} b_{j} \sum_{i=1}^{m} p^{-i}\left\|W_{j}^{((k-l+2) m-i)}\right\|^{2}+q^{k+2} \Delta .
$$

By induction we can prove from the above inequality that (6.16) holds for every $k \geq 0$. This completes the proof.

Remark 6.7. It is easy to verify the following inequality,

$$
0<q+q r \leq \max \left(q, \frac{\beta}{-(2 \alpha+\beta)}\right) .
$$

Hence, $q+q r<1$ if $\alpha+\beta<0$ and the right-hand side of (6.16) goes to zero for increasing $k$.

Remark 6.8. If condition (6.15) is replaced by the weaker condition

$$
2 \alpha+\beta<0,
$$

then Theorem 6.6 is still valid. Then, however, it is not guaranteed that $q+q r<1$.

COROLlary 6.9. Under the assumptions of Theorem 6.6, inequality (6.16) implies that for every $n \geq m$

$$
\sum_{j=1}^{s} b_{j}\left\|W_{j}^{(n)}\right\|^{2} \leq(q+q r)^{n / m} \Delta .
$$

Proof. It follows from (6.16) that for every $k \geq 0, i \in\{1, \ldots, m\}$,

$$
\sum_{j=1}^{s} b_{j}\left\|W_{j}^{((k+2) m-i)}\right\|^{2} \leq p^{i} q^{2}(q+q r)^{k} \Delta=q^{2-i / m}(q+q r)^{k} \Delta \leq(q+q r)^{k+2-i / m} \Delta,
$$

which gives (6.18). 
TheOREM 6.10. Suppose that the method $(A, b, c)$ with a nonsingular matrix $A$ is algebraically stable, $b_{i}>0$ for all $i$ and (6.15) holds. Then there exists a constant $C_{7}$, depending only on the coefficients of the method, such that for every $n \geq m$,

$$
\left\|w_{n+1}\right\| \leq|R(\infty)|^{n+1-m}\left\|w_{m}\right\|+C_{7}(q+q r)^{1 / 2} \Delta^{1 / 2} \psi_{n+1-m}\left(|R(\infty)|,(q+q r)^{1 / 2 m}\right),
$$

where the function $\psi_{n}(x, y)$ is defined by (4.13).

Proof. Considering (5.16) and the assumptions of the theorem, there exists a constant $C_{7}$, depending only on the coefficients, such that

$$
\left\|w_{n+1}\right\| \leq \mid R(\infty)\|\| w_{n} \|+C_{7}\left(\sum_{j=1}^{s} b_{j}\left\|W_{j}^{(n)}\right\|^{2}\right)^{1 / 2},
$$

which in combination with (6.18) gives

$$
\begin{aligned}
\left\|w_{n+1}\right\| \leq & |R(\infty)|\left\|w_{n}\right\|+C_{7}(q+q r)^{n / 2 m} \Delta^{1 / 2} \\
\leq & |R(\infty)|^{n+1-m}\left\|w_{m}\right\| \\
& +C_{7}(q+q r)^{1 / 2} \Delta^{1 / 2} \sum_{i=0}^{n-m}|R(\infty)|^{i}(q+q r)^{(n-m-i) / 2 m} .
\end{aligned}
$$

This implies the result of the theorem.

Corollary 6.11. Suppose (6.15) holds. Then for any Radau IA, Radau IIA, or Lobatto IIIC method, there exists a constant $C_{7}$ depending only on the coefficients of the method, such that for every $n \geq m$,

$$
\left\|w_{n+1}\right\| \leq C_{7}(q+q r)^{n / 2 m} \Delta^{1 / 2} .
$$

Corollary 6.12. Suppose (6.15) holds. Then for any Gauss method there exists a constant $C_{7}$, depending only on the coefficients of the method, such that for every $n \geq m$,

$$
\left\|w_{n+1}\right\| \leq\left\|w_{m}\right\|+\frac{C_{7}(q+q r)^{1 / 2} \Delta^{1 / 2}}{1-(q+q r)^{1 / 2 m}}
$$

Remark 6.13. It is easy to extend the results of this section to the case of $(k, l)$ algebraically stable methods if we impose some restrictions on stepsize similar to those in [15].

7. Extension to a more general class of equations. In this section, we generalize some stability results to the more general equations

$$
\left\{\begin{array}{l}
y^{\prime}(t)=L(t) y(t)+M(t) y(t-\tau(t))+N(t) y^{\prime}(t-\tau(t)), \quad t \geq t_{0} \\
y(t)=g(t), \quad t \leq t_{0}
\end{array}\right.
$$

and

$$
\left\{\begin{array}{l}
y^{\prime}(t)=f\left(t, y(t), y(t-\tau(t)), y^{\prime}(t-\tau(t))\right), \quad t \geq t_{0} \\
y(t)=g(t), \quad t \leq t_{0}
\end{array}\right.
$$

The ideas are related to those in [13]. We assume that there exists a constrained mesh in the interval $\left[t_{0},+\infty\right)$ such that

$$
t_{n}-\tau\left(t_{n}\right)=t_{n-m}, \quad n \geq m,
$$


for some integer $m$. The corresponding discretized schemes for (7.1) and (7.2) are (3.5-3.7) and (2.4-2.6), respectively. Then, a similar analysis leads to the following general results.

TheOREM 7.1. Suppose that the method $(A, b, c)$ with a nonsingular matrix $A$ is strictly stable at infinity and that the coefficient matrices of (7.1) satisfy

$$
\lim _{n \rightarrow \infty} h_{n}^{-1}\left\|L^{-1}\left(t_{n}+c_{i} h_{n}\right)\right\|=0, \quad \lim _{n \rightarrow \infty} h_{n-m}^{-1}\left\|L^{-1}\left(t_{n}+c_{i} h_{n}\right) N\left(t_{n}+c_{i} h_{n}\right)\right\|=0,
$$

and

$$
\limsup _{n \rightarrow \infty}\left\|L^{-1}\left(t_{n}+c_{i} h_{n}\right) M\left(t_{n}+c_{i} h_{n}\right)\right\|=k_{0}<1,
$$

for $i=1,2, \ldots, s$. Then, the scheme (3.5-3.7) is asymptotically stable for (7.1).

THEOREM 7.2. Suppose that the method $(A, b, c)$ with a nonsingular matrix $A$ is strictly stable at infinity and that the function $f$ of (7.2) satisfies conditions (5.2) and (5.3) with

$$
\lim _{n \rightarrow \infty} h_{n} \alpha\left(t_{n}+c_{i} h_{n}\right)=-\infty, \quad \lim _{n \rightarrow \infty} \frac{\gamma\left(t_{n}+c_{i} h_{n}\right)}{h_{n-m} \alpha\left(t_{n}+c_{i} h_{n}\right)}=0,
$$

and

$$
\limsup _{n \rightarrow \infty} \frac{\beta\left(t_{n}+c_{i} h_{n}\right)}{\left|\alpha\left(t_{n}+c_{i} h_{n}\right)\right|}=k_{2}<1,
$$

for $i=1,2, \ldots, s$. Then, the scheme (2.4-2.6) is asymptotically stable for (7.2), i.e., (5.13) and (5.14) hold.

Remark 7.3. For the constant delay system (1.3), we can consider a constant stepsize strategy, i.e., $h_{n}=-\log q / m$, such that (7.3), (7.4), and (7.5) hold if $\rho\left[L^{-1} M\right]<1$.

Remark 7.4. If the delay $\tau(t)$ satisfies the conditions

$$
\begin{gathered}
0<\tau_{0} \leq \tau(t)<t, \quad t \geq t_{0}, \\
0<q_{*} \leq 1-\tau^{\prime}(t) \leq q^{*}<1, \quad t \geq t_{0},
\end{gathered}
$$

then $t-\tau(t)$ is strictly increasing and $\lim _{t \rightarrow \infty} t-\tau(t)=+\infty$, which guarantees the existence of a constrained mesh (cf. $[5,13])$ and

$$
h_{n-m}=h_{n}-\left(\tau\left(t_{n+1}\right)-\tau\left(t_{n}\right)\right) \leq q^{*} h_{n} .
$$

Hence,

$$
\lim _{n \rightarrow \infty} h_{n}=+\infty
$$

which implies (7.4) if $L^{-1}(t)$ and $N(t)$ are bounded. In the case of vanishing delays, i.e., $\tau(0)=0$, we can choose an appropriate point $t_{0}>0$ and assume we know an approximate solution in $\left[0, t_{0}\right]$ such that $(7.8)$ hold.

Remark 7.5. If $c_{i} \in\{0,1\}$ or $\tau(t)$ is of the form $q t+d$, we can appropriately choose stepsize such that (7.3) implies

$$
t_{n}+c_{i} h_{n}-\tau\left(t_{n}+c_{i} h_{n}\right)=t_{n-m}+c_{i} h_{n-m},
$$

see [5, section 6.3]. In the other case, however, the above equality may no longer hold, an interpolation for the delay argument is possibly necessary and a rigorous theoretical analysis is missing (and outside the scope of the present paper). 
8. Concluding remarks. In this work the stability of Runge-Kutta methods for both linear and nonlinear nonautonomous pantograph equations has been analyzed. A new approach has been introduced to derive the asymptotic stability of numerical methods and some sufficient and necessary conditions have been found. By further exploiting the special structure of the stepsize, we have also obtained some upper bounds for the error growth.

The techniques of this paper can be applied to investigate the stability of numerical methods for the constant delay system derived from the pantograph equation. They could also possess a potential applicability to integro-differential equations with proportional delays.

Acknowledgments. The authors are grateful to the anonymous referee for his valuable comments and remarks. They are also indebted to Professor Hermann Brunner for suggesting the extension of the study to a more general class of equations.

\section{REFERENCES}

[1] C. T. H. Baker, Retarded differential equations, J. Comput. Appl. Math., 125 (2000), pp. 309335.

[2] A. Bellen, N. Guglielmi, and L. Torelli, Asymptotic stability properties of $\theta$-methods for the pantograph equation, Appl. Numer. Math., 24 (1997), pp. 279-293.

[3] A. Bellen, N. Guglielmi, And M. Zennaro, Numerical stability of nonlinear delay differential equations of neutral type, J. Comput. Appl. Math., 125 (2000), pp. 251-263.

[4] A. Bellen, S. Maset, And L. Torelli, Contractive initializing methods for the pantograph equation of neutral type, Recent Trends in Numerical Analysis, 3 (2000), pp. 35-41.

[5] A. Bellen and M. Zennaro, Numerical Methods for Delay Differential Equations, Oxford University Press, Oxford, UK, 2003.

[6] M. D. Buhmann And A. Iserles, Numerical analysis of functional equations with a variable delay, in Numerical Analysis 1991 (Dundee, 1991), D. F. Griffiths and G. A. Watson, eds., Longman, Sci. Tech., Harlow, UK, 1992, pp. 17-33.

[7] M. D. Buhmann And A. Iserles, On the dynamics of a discretized neutral equation, IMA J. Numer. Anal., 12 (1992), pp. 339-363.

[8] M. D. Buhmann AND A. IsERLEs, Stability of the discretized pantograph differential equation, Math. Comp., 60 (1993), pp. 575-589.

[9] M. D. Buhmann, A. Iserles, And S. P. Norsett, Runge-Kutta methods for neutral differential equations, in Contributions in Numerical Mathematics, World Sci. Ser. Appl. Anal. 2, World Scientific, River Edge, NJ, 1993, pp. 85-98.

[10] K. Burrage and J. C. Butcher, Stability criteria for implicit Runge-Kutta methods, SIAM J. Numer. Anal., 16 (1979), pp. 46-57.

[11] K. Burrage and J. C. Butcher, Nonlinear stability of a general class of differential equation methods, BIT, 20 (1980), pp. 185-203.

[12] K. Dekker And J. G. Verwer, Stability of Runge-Kutta Methods for Stiff Nonlinear Differential Equations, CWI Monographs 2, North-Holland, Amsterdam, 1984.

[13] N. Guglielmi and M. Zennaro, Stability of one-leg $\Theta$-methods for the variable coefficient pantograph equation on the quasi-geometric mesh, IMA J. Numer. Anal., 23 (2003), pp. 421438.

[14] E. Hairer and G. Wanner, Solving Ordinary Differential Equations. Stiff and DifferentialAlgebraic Problems II, Springer-Verlag, Berlin, 1991.

[15] C. Huang, H. Fu, S. Li, And G. Chen, Stability analysis of Runge-Kutta methods for nonlinear delay differential equations, BIT, 39 (1999), pp. 270-280.

[16] A. IsERLES, On the generalized pantograph functional-differential equations, European J. Appl. Math., 4 (1993), pp. 1-38.

[17] A. Iserles, Numerical analysis of delay differential equations with variable delays, Ann. Numer. Math., 1 (1994), pp. 133-152.

[18] A. IserLes, Exact and discretized stability of the pantograph equation, Appl. Numer. Math., 24 (1997), pp. 295-308.

[19] A. Iserles and J. TerJeki, Stability and asymptotic stability of functional-differential equations, J. London Math. Soc. (2), 51 (1995), pp. 559-572. 
[20] Z. JACKIEwICZ, Asymptotic stability analysis of $\theta$-methods for functional differential equations, Numer. Math., 43 (1984), pp. 389-396.

[21] T. Кото, Stability of Runge-Kutta methods for the generalized pantograph equation, Numer. Math., 84 (1999), pp. 233-247.

[22] Y. LIU, Stability analysis of $\theta$-methods for neutral functional-differential equations, Numer. Math., 70 (1995), pp. 473-485.

[23] Y. LiU, Asymptotic behaviour of functional-differential equations with proportional time delay, European J. Appl. Math., 7 (1996), pp. 11-30.

[24] Y. Liu, On the $\theta$-methods for delay differential equations with infinite lag, J. Comput. Appl. Math., 71 (1996), pp. 177-190.

[25] Y. LiU, Numerical investigation of the pantograph equation, Appl. Numer. Math., 24 (1997), pp. 309-317.

[26] R. Vermiglio and L. Torelli, A stable numerical approach for implicit non-linear neutral delay differential equations, BIT, 43 (2003), pp. 195-C215.

[27] M. Zennaro, Asymptotic stability analysis of Runge-Kutta methods for nonlinear systems of delay differential equations, Numer. Math., 77 (1997), pp. 549-563.

[28] C. J. Zhang And G. Sun, Nonlinear stability of variable stepsize Runge-Kutta methods applied to infinite delay-differential equations, Math. Comput. Modelling, 39 (2004), pp. 495-503.

[29] C. J. Zhang And G. Sun, The discrete dynamics of nonlinear infinite-delay-differential equations, Appl. Math. Lett., 15 (2002), pp. 521-526. 